\title{
MLFMA Solutions of Transmission Problems Involving Realistic Metamaterial Walls
}

\author{
Özgür Ergül ${ }^{1,2}$, Alper Ünal ${ }^{2}$, and Levent Gürel ${ }^{1,2}$ \\ ${ }^{1}$ Department of Electrical and Electronics Engineering \\ ${ }^{2}$ Computational Electromagnetics Research Center (BiLCEM) \\ Bilkent University, TR-06800, Bilkent, Ankara, Turkey \\ ergul@ee.bilkent.edu.tr, runal@ug.bilkent.edu.tr, lgurel@bilkent.edu.tr
}

\begin{abstract}
We present the solution of multilayer metamaterial (MM) structures containing large numbers of unit cells, such as split-ring resonators. Integral-equation formulations of scattering problems are solved iteratively by employing a parallel implementation of the multilevel fast multipole algorithm. Due to ill-conditioned nature of the problems, advanced preconditioning techniques are used to obtain rapid convergence in the iterative solutions. By constructing a sophisticated simulation environment, we accurately and efficiently investigate large and complicated MM structures.
\end{abstract}

\section{INTRODUCTION}

After they were first fabricated in 2000 [1], metamaterials (MMs) have attracted a great amount of interest in the scientific community due to their useful electromagnetic properties. These structures are usually constructed by periodically arranging unit cells in a host medium. In this paper, we specifically investigate MMs that contain split-ring resonators (SRRs) as basic building blocks. We do not assume any periodicity or homogeneity for these structures. We take into account that MMs are actually of finite extent, they exhibit interface properties, and their unit cells (SRRs) are interacting with each other. Our accurate simulations transform into large computational problems, which require special acceleration methods, such as the multilevel fast multipole algorithm (MLFMA) [2].

Fig. 1 presents the SRR geometry and its discretization used in our simulations. When the host medium has a relative

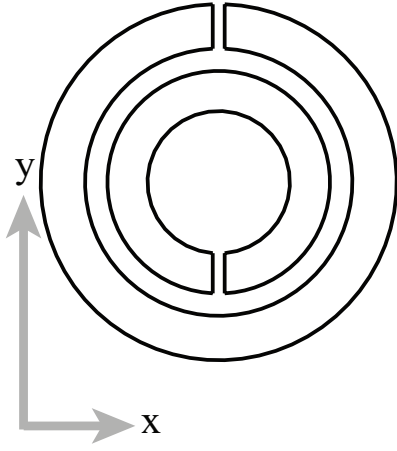

(a)

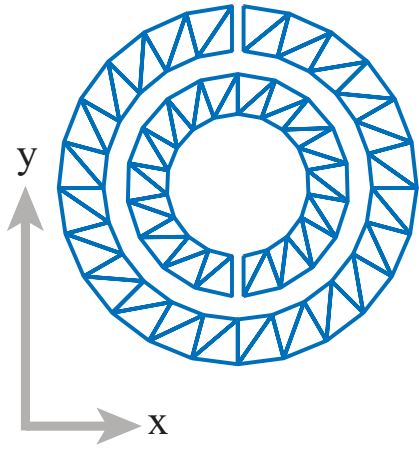

(b)
Fig. 1. (a) An SRR geometry used in our simulations. (b) Discretization of the SRR with 84 triangles corresponding to 82 unknowns.

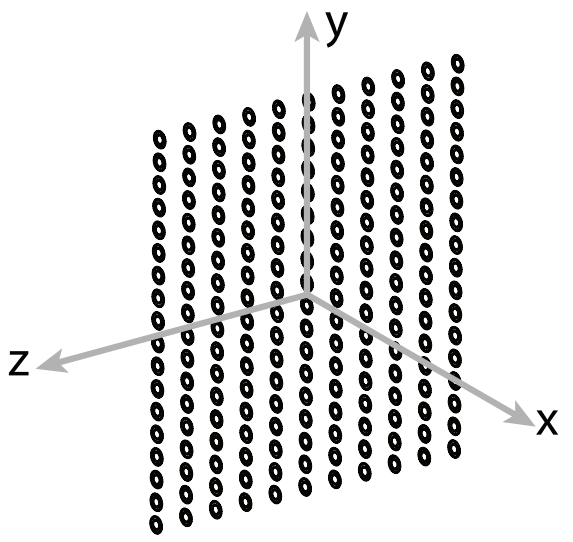

(a)

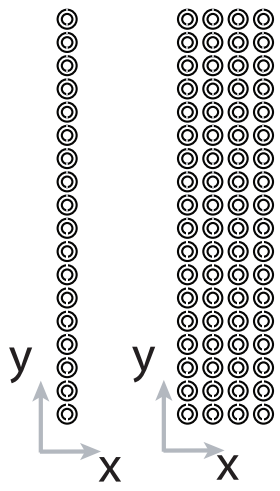

(b)
Fig. 2. An SRR array (1-layer MM wall). (b) Side views of 1-layer and 4-layer MM walls.

permittivity of 4.8 similar to the experimental case [3], this SRR resonates around $100 \mathrm{GHz}$ [3]-[6]. In our simulation environment, SRRs are modeled by using perfectly-conducting surfaces with zero thickness. Then, the electric-field integral equation (EFIE) is employed to formulate the scattering problems. However, matrix equations produced by EFIE are usually ill-conditioned and their iterative solutions are difficult [7]. In our case, quick convergence of the iterations is further inhibited by the resonant nature of MMs. Therefore, we employ advanced preconditioning techniques for the efficient solutions of MM problems.

Fig. 2 presents some examples of MM structures constructed by using SRRs. As depicted in Fig. 2(a), we arrange SRRs as a two-dimensional array in the $z-y$ plane. In addition, we construct multilayer MM walls as shown in Fig. 2(b) by stacking the layers in the $x$ direction. Periodicity of the unit cells are $263 \mu \mathrm{m}, 263 \mu \mathrm{m}$, and $450 \mu \mathrm{m}$ in the $x, y$, and $z$ directions, respectively.

\section{EFIE Formulation OF METAMATERIAL PROBLEMS}

For perfectly-conducting objects, EFIE can be written directly from the boundary condition for the tangential electric 

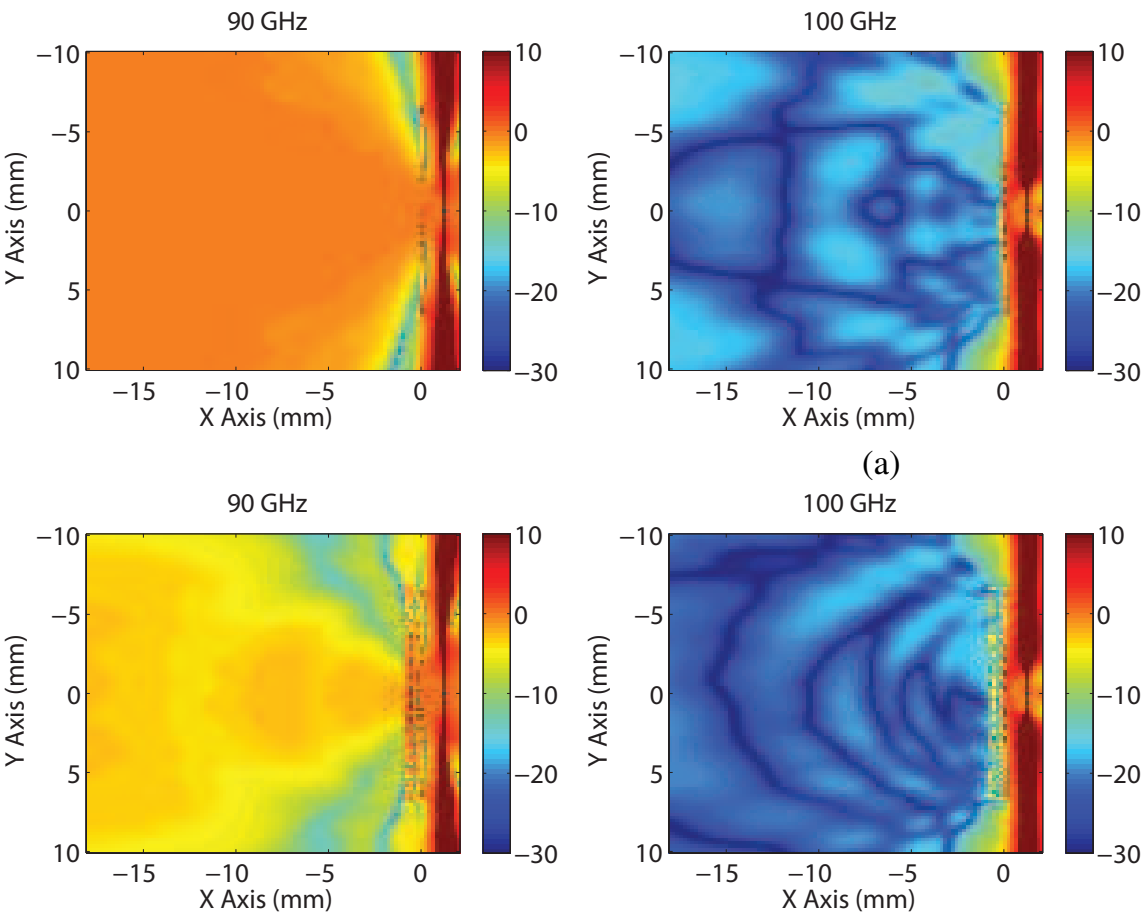

(b)
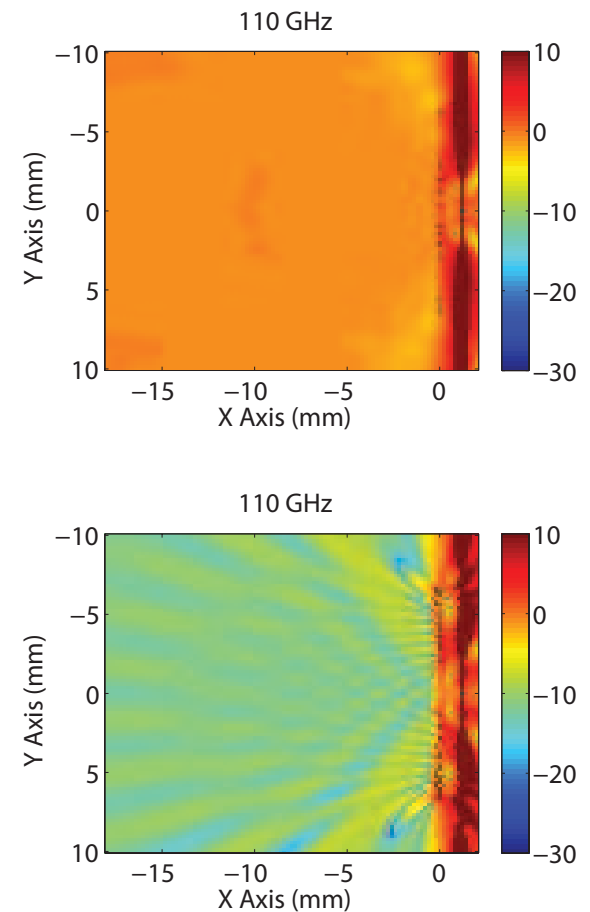

Fig. 3. Power transmission for (a) 1-layer and (b) 4-layer SRR walls. Each layer contains $51 \times 29$ SRRs. A shadowing effect is observed at $100 \mathrm{GHz}$ due to an effective negative permeability induced in the medium.

field (in phasor notation with the $e^{-i \omega t}$ convention) as

$\hat{\boldsymbol{t}} \cdot i k \eta \int_{S} d \boldsymbol{r}^{\prime}\left[\boldsymbol{J}\left(\boldsymbol{r}^{\prime}\right)+\frac{1}{k^{2}} \nabla^{\prime} \cdot \boldsymbol{J}\left(\boldsymbol{r}^{\prime}\right) \nabla\right] g\left(\boldsymbol{r}, \boldsymbol{r}^{\prime}\right)=-\hat{\boldsymbol{t}} \cdot \boldsymbol{E}^{i}(\boldsymbol{r})$

by expressing the scattered electric field in terms of the induced (unknown) surface current $\boldsymbol{J}(\boldsymbol{r})$. In (1), $\hat{\boldsymbol{t}}$ is any tangential unit vector on the surface at the observation point $\boldsymbol{r}, \boldsymbol{E}^{i}(\boldsymbol{r})$ is the incident electric field, $k=\omega \sqrt{\mu \epsilon}$ is the wavenumber, $\eta=\sqrt{\mu / \epsilon}$ is the impedance of the host medium, and

$$
g\left(\boldsymbol{r}, \boldsymbol{r}^{\prime}\right)=\frac{e^{i k R}}{4 \pi R} \quad\left(R=\left|\boldsymbol{r}-\boldsymbol{r}^{\prime}\right|\right)
$$

denotes the homogenous-space Green's function.

For numerical solutions of MM problems, discretization of EFIE leads to $N \times N$ dense matrix equations as

$$
\sum_{n=1}^{N} Z_{m n}^{E} a_{n}=v_{m}^{E}, \quad m=1, \ldots, N
$$

where the matrix elements and the elements of the excitation vector are derived as

$$
\begin{aligned}
Z_{m n}^{E} & =i k \eta \int_{S_{m}} d \boldsymbol{r} \boldsymbol{t}_{m}(\boldsymbol{r}) \cdot \int_{S_{n}} d \boldsymbol{r}^{\prime} g\left(\boldsymbol{r}, \boldsymbol{r}^{\prime}\right) \cdot \boldsymbol{b}_{n}\left(\boldsymbol{r}^{\prime}\right) \\
& +\frac{i \eta}{k} \int_{S_{m}} d \boldsymbol{r} \boldsymbol{t}_{m}(\boldsymbol{r}) \cdot \int_{S_{n}} d \boldsymbol{r}^{\prime} \nabla^{\prime} \cdot \boldsymbol{b}_{n}\left(\boldsymbol{r}^{\prime}\right) \nabla g\left(\boldsymbol{r}, \boldsymbol{r}^{\prime}\right)
\end{aligned}
$$

and

$$
v_{m}^{E}=-\int_{S_{m}} d \boldsymbol{r} \boldsymbol{t}_{m}(\boldsymbol{r}) \cdot \boldsymbol{E}^{i}(\boldsymbol{r})
$$

respectively. In (4) and (5), $S_{m}$ and $S_{n}$ symbolize the spatial supports of the $m$ th testing function $\boldsymbol{t}_{m}(\boldsymbol{r})$ and $n$th basis function $\boldsymbol{b}_{n}\left(\boldsymbol{r}^{\prime}\right)$, respectively. We apply a Galerkin scheme and choose the basis and testing functions as Rao-WiltonGlisson (RWG) functions defined on planar triangles [8]. The matrix equation in (3) is solved iteratively, and the matrixvector products are accelerated by MLFMA.

Following the solution of the coefficients $a_{n}$, we obtain the scattered electric and magnetic fields as

$$
\begin{aligned}
\boldsymbol{E}^{S}(\boldsymbol{r}) & =i k \eta \sum_{n=1}^{N} \int_{S_{n}} d \boldsymbol{r}^{\prime} g\left(\boldsymbol{r}, \boldsymbol{r}^{\prime}\right) \boldsymbol{b}_{n}\left(\boldsymbol{r}^{\prime}\right) a_{n} \\
& +\frac{i \eta}{k} \sum_{n=1}^{N} \int_{S_{n}} d \boldsymbol{r}^{\prime} \nabla^{\prime} \cdot \boldsymbol{b}_{n}\left(\boldsymbol{r}^{\prime}\right) \nabla g\left(\boldsymbol{r}, \boldsymbol{r}^{\prime}\right) a_{n}
\end{aligned}
$$

and

$$
\boldsymbol{H}^{s}(\boldsymbol{r})=\sum_{n=1}^{N} \int_{S_{n}} d \boldsymbol{r}^{\prime} \boldsymbol{b}_{n}\left(\boldsymbol{r}^{\prime}\right) \times \nabla^{\prime} g\left(\boldsymbol{r}, \boldsymbol{r}^{\prime}\right) a_{n},
$$

respectively. Then, the average power density at an observation point $r$ is calculated as

$$
\boldsymbol{P}_{a v}^{t}(\boldsymbol{r})=\left\langle\boldsymbol{P}^{t}(\boldsymbol{r})\right\rangle=\frac{1}{2} \operatorname{Re}\left\{\boldsymbol{E}^{t}(\boldsymbol{r}) \times\left[\boldsymbol{H}^{t}(\boldsymbol{r})\right]^{*}\right\},
$$

where $\boldsymbol{E}^{t}(\boldsymbol{r})=\boldsymbol{E}^{i}(\boldsymbol{r})+\boldsymbol{E}^{s}(\boldsymbol{r})$ and $\boldsymbol{H}^{t}(\boldsymbol{r})=\boldsymbol{H}^{i}(\boldsymbol{r})+\boldsymbol{H}^{s}(\boldsymbol{r})$ are the total electric and magnetic fields, respectively. For a 


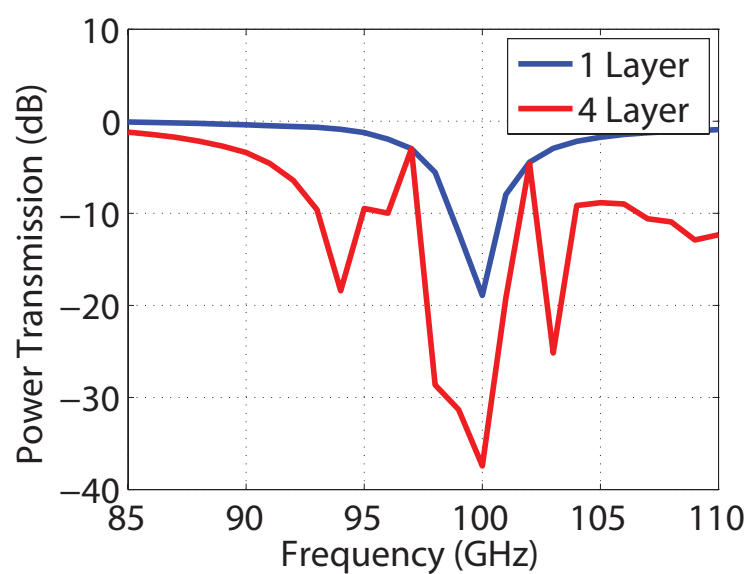

Fig. 4. Power transmission for 1-layer and 4-layer SRR walls as a function of frequency.

MM wall, we define the power transmission as

$$
\tau(\boldsymbol{r})=\frac{\left|\boldsymbol{P}_{a v}^{t}(\boldsymbol{r})\right|}{\left|\boldsymbol{P}_{a v}^{i}(\boldsymbol{r})\right|},
$$

where

$$
\boldsymbol{P}_{a v}^{i}(\boldsymbol{r})=\left\langle\boldsymbol{P}^{i}(\boldsymbol{r})\right\rangle=\frac{1}{2} \operatorname{Re}\left\{\boldsymbol{E}^{i}(\boldsymbol{r}) \times\left[\boldsymbol{H}^{i}(\boldsymbol{r})\right]^{*}\right\}
$$

is the average incident power density due to external sources.

\section{NUMERICAL RESULTS}

Fig. 3 demonstrates the power transmission results for 1layer and 4-layer SRR walls at 90, 100, and $110 \mathrm{GHz}$. Each layer of the walls includes $51 \times 29$ SRRs. Power transmission is calculated on the $z=0$ plane and SRR walls are located at $x=0$. The source is a Hertzian dipole oriented in the $y$ direction and located at $x=1.2 \mathrm{~mm}$. Transmission region is on the left of the MM walls. Fig. 3(a) shows that the power transmission through the 1-layer wall is close to unity corresponding to 0 decibels $(\mathrm{dB})$ at 90 and $110 \mathrm{GHz}$. In other words, the MM wall is transparent at these frequencies and the fields radiated from the dipole are transmitted through the structure. However, at $100 \mathrm{GHz}$, the wall becomes opaque and the transmitted power drops significantly. This is due to effective negative permeability induced in the medium, which is triggered by a physical resonance of the SRR structure.

When the number of layers increases, transmission properties of the MM structures change significantly. Especially coupling among the layers leads to more oscillatory power transmission with respect to frequency. In addition, bandwidth of the resonance tends to increase with the inclusion of more layers. As an example, Fig. 3(b) presents the transmission results for the $4 \times 51 \times 29$ SRR wall. In addition to the shadowing at $100 \mathrm{GHz}$, we also observe relatively lower transmission at 90 and $110 \mathrm{GHz}$ compared to the 1-layer results in Fig. 3(a). For a more quantitative comparison, Fig. 4 presents the power transmission calculated at a single point $(x=-1.2 \mathrm{~mm})$ as a function of frequency for the 1-layer and 4-layer structures.

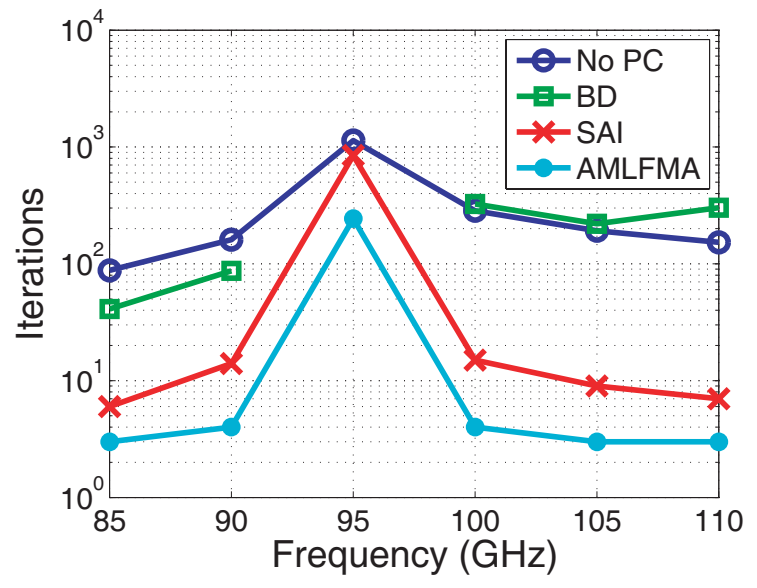

Fig. 5. Number of GMRES iterations for the solution of a scattering problem involving a $1 \times 51 \times 29$ SRR wall. Using a BD preconditioner, convergence cannot be achieved within 4000 iterations at $95 \mathrm{GHz}$.

Increasing the number of layers, power transmission becomes oscillatory and the stop-band region is not confined to a narrow band of frequencies around $100 \mathrm{GHz}$.

Fig. 5 presents the iteration counts for the solution of the scattering problem involving the $1 \times 51 \times 29$ SRR wall. For a range of frequencies from $85 \mathrm{GHz}$ to $110 \mathrm{GHz}$, iterative solutions are performed by a generalized-minimal-residual (GMRES) algorithm with no restart. We observe that the solution of the problem is extremely difficult at $95 \mathrm{GHz}$ due to a numerical resonance of the MM structure. At this frequency, residual error can be reduced below $10^{-3}$ with more than 1000 iterations without a preconditioner. Using a block-diagonal (BD) preconditioner accelerates the iterative solutions slightly for low frequencies, where the diagonal blocks involve large numbers of matrix elements. However, $\mathrm{BD}$ preconditioner becomes useless for higher frequencies. At the resonance frequency $(95 \mathrm{GHz})$, using $\mathrm{BD}$ preconditioner is even worse than the no-preconditioner case; convergence cannot be achieved within 4000 iterations. By employing a sparse-approximate-inverse (SAI) preconditioner, iterative solutions are accelerated significantly, except for the resonance frequency. We note that this acceleration is provided at the cost of the increased setup time for the construction of the preconditioner [9]. Finally, to further accelerate the iterations, we employ a nested preconditioning scheme based on a flexible GMRES algorithm and an approximate MLFMA (AMLFMA) preconditioner [10]. Using this highly effective preconditioner, the number of iterations is only 244 at the resonance frequency, while it is less than 5 for ordinary frequencies.

Finally, Fig. 6 presents the transmission results for very large MM walls involving 20 layers. The scattering problems are solved by MLFMA parallelized into 64 processes running on a cluster of quad-core Intel Xeon processors connected via an Infiniband network. Fig. 6(a) shows the power transmission for a $20 \times 18 \times 11$ wall, which is discretized with 324,720 unknowns. In Fig. 6(b), power transmission is depicted for 


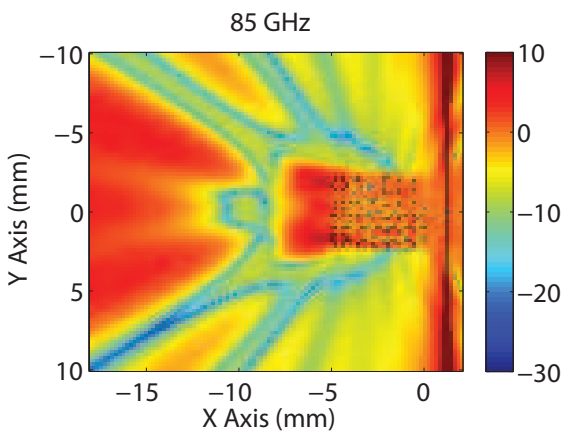

$85 \mathrm{GHz}$

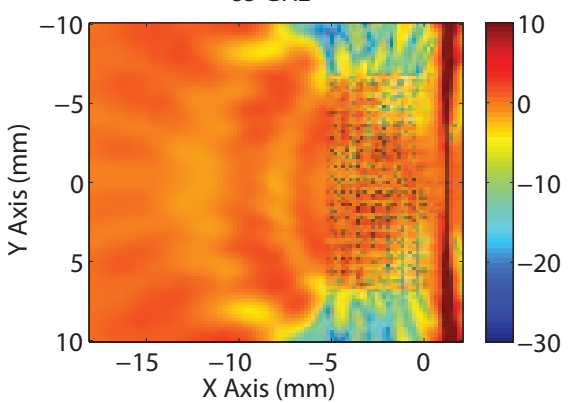

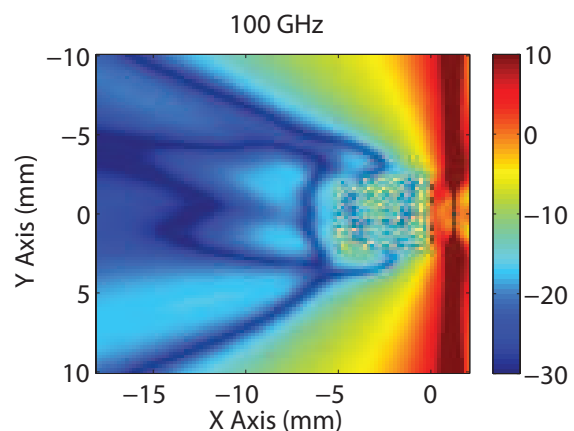

(a)

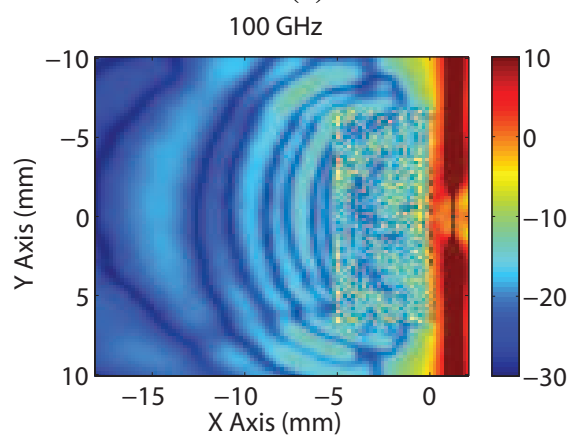

$110 \mathrm{GHz}$

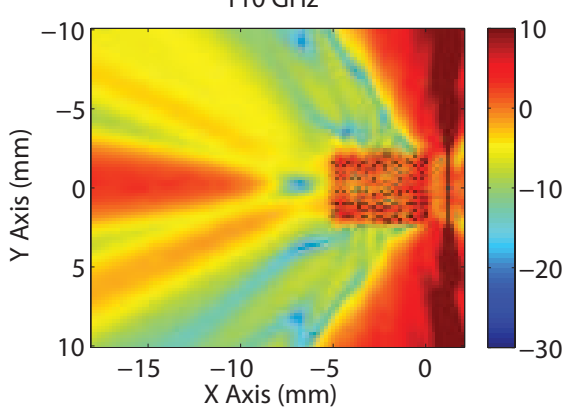

$110 \mathrm{GHz}$

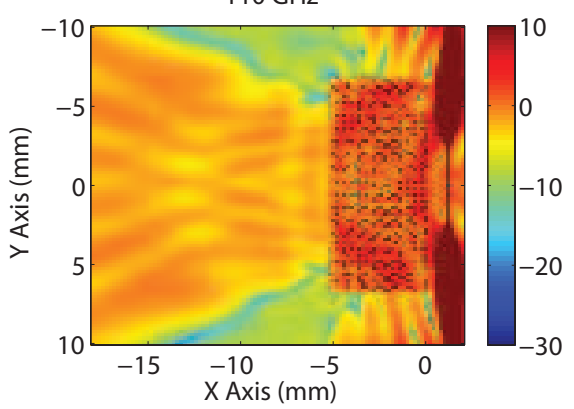

(b)

Fig. 6. Transmission results for 20 -layer SRR walls. Each layer involves (a) $18 \times 11$ and (b) $51 \times 29$ SRRs. A shadowing effect is observed at 100 GHz due to effective negative permeability induced in the medium.

a $20 \times 51 \times 29$ wall, which is discretized with $2,425,560$ unknowns.

\section{CONCLUSions}

We report our efforts for the fast and accurate solution of large MM structures constructed by SRRs. Scattering problems are formulated with EFIE and the resulting dense matrix equations are solved iteratively by MLFMA. We employ robust preconditioning techniques to obtain rapid convergence for the iterative solutions. By constructing a sophisticated simulation environment, we are able to investigate the transmission properties of realistic MM structures involving large numbers of unit cells.

\section{ACKNOWLEDGMENT}

This work was supported by the Scientific and Technical Research Council of Turkey (TUBITAK) under Research Grants 105E172 and 107E136, by the Turkish Academy of Sciences in the framework of the Young Scientist Award Program (LG/TUBA-GEBIP/2002-1-12), and by contracts from ASELSAN and SSM.

\section{REFERENCES}

[1] D. R. Smith, W. J. Padilla, D. C. Vier, S. C. Nemat-Nasser, and S. Schultz, "Composite Medium with Simultaneously Negative Permeability and Permittivity," Phys. Rev. Lett., vol. 84, 4184, 2000.

[2] J. Song, C.-C. Lu, and W. C. Chew, "Multilevel fast multipole algorithm for electromagnetic scattering by large complex objects," IEEE Trans. Antennas Propagat., vol. 45, no. 10, pp. 1488-1493, Oct. 1997.

[3] M. Gokkavas, K. Güven, I. Bulu, K. Aydın, R. S. Penciu, M. Kafesaki, C. M. Soukoulis, and E. Özbay, "Experimental demonstration of a lefthanded metamaterial operating at $100 \mathrm{GHz}$," Phys. Rev. B., vol. 73, no. 193103, 2006.

[4] Ö. Ergül, A. Ünal, and L. Gürel, "Accurate modeling of metamaterials with MLFMA," in Proc. European Conference on Antennas and Propagation (EuCAP), 350094, 2006.

[5] Ö. Ergül, Ç. Yavuz, A. Ünal, and L. Gürel, "Investigation of various metamaterial structures using multilevel fast multipole algorithm," in Proc. IEEE Antennas and Propagation Soc. Int. Symp., 2007, pp. 18451848.

[6] L. Gürel, Ö. Ergül, and A. Ünal, "Accurate analysis of metamaterials involving finite arrays of split-ring resonators and thin wires," in Proc. Progress in Electromagnetics Research Symposium (PIERS), 2007, pp. 470-473.

[7] L. Gürel and Ö. Ergül, "Comparisons of FMM implementations employing different formulations and iterative solvers," in Proc. IEEE Antennas and Propagation Soc. Int. Symp., vol. 1, 2003, pp. 19-22.

[8] S. M. Rao, D. R. Wilton, and A. W. Glisson, "Electromagnetic scattering by surfaces of arbitrary shape," IEEE Trans. Antennas Propagat., vol. AP-30, no. 3, 409-418, May. 1982.

[9] Y. Saad, Iterative Methods for Sparse Linear Systems. Philadelphia: SIAM, 2003.

[10] T. Malas, Ö. Ergül, and L. Gürel, "Approximate MLFMA as an efficient preconditioner," in Proc. IEEE Antennas and Propagation Soc. Int. Symp., 2007, pp. 1289-1292. 